\title{
The last dance of the bashful ballerina?^
}

\author{
K. Mursula and I. Virtanen
}

Department of Physics, PO Box 3000, 90014 University of Oulu, Finland
e-mail: kalevi .mursula@oulu. fi

Received 27 December 2009 / Accepted 22 June 2010

\begin{abstract}
Aims. The heliospheric magnetic field (HMF) has long been hemispherically asymmetric so that the field in the northern hemisphere is weaker and the area larger than in the south. This asymmetry, also called the bashful ballerina, has existed during roughly three-year intervals of the late declining to minimum phase of solar cycles 16-22. We study the HMF and its hemispheric asymmetry during the exceptional solar cycle 23 .

Methods. We use NASA National Space Science Data Center OMNI database, which contains all solar wind and HMF observations at the Earth's orbit, and coronal field predictions by Wilcox Solar Observatory. We present a new method to study the global hemispheric asymmetry by using the power $n$ of the radial decrease of the radial field from the coronal source surface to 1 AU.

Results. We find that the HMF is exceptional at low latitudes in solar cycle 23: while the typical latitudinal variation was attained in the north in 2008, it did not take place in the south until Spring 2009. Thus, the Rosenberg-Coleman rule is abnormally delayed or broken for the first time in 50 years. The $n$-values verify the clear northern dominance in cycles $21-22$. However, the low-latitude observations depict a considerably smaller asymmetry in cycle 23, although Ulysses observations at high latitudes show an equally large asymmetry in 2007 and in 1994-1995. We argue that the weak low-latitude visibility of the asymmetry in cycle 23 is due to the exceptionally weak polar fields, leading to large tilt angle and a wide current sheet.

Conclusions. We note that the exceptional properties of cycle 23 (weak dynamo, large tilt, small asymmetry) agree with the long-term evolution of hemispheric asymmetry viewed at the Earth. The active Sun is seen as more asymmetric at the Earth than the quiet Sun because the polar coronal holes with unipolar fields extend closer to the equator, allowing their asymmetry to be viewed even at low latitudes. We suggest that, after the period of weak activity and small asymmetry at 1 AU that started with cycle 23, the hemispheric asymmetry will again, with the increasingly active cycles, become better visible at 1 AU but the asymmetry will be oppositely oriented, including a northward shifted current sheet, and larger areas but weaker intensities in the south. Thus, the ballerina should no longer be systematically bashful for some $100-150$ years.
\end{abstract}

Key words. Sun: heliosphere - Sun: activity - Sun: corona - solar wind

\section{Introduction}

Long-term HMF observations have depicted interesting systematic hemispheric asymmetries that have important practical consequences and far-reaching implications for the understanding of solar magnetism. It was noted that the heliospheric current sheet (HCS) was shifted southwards by several degrees during the first fast latitude scan of the Ulysses probe in 1994-1995 (Simpson et al. 1996; Crooker et al. 1997; Smith et al. 2000). Subsequently, a general pattern was identified (Mursula \& Hiltula 2003) using long-term HMF observations at $1 \mathrm{AU}$, according to which the HMF sector prevalent in the northern solar hemisphere dominates the HMF sector occurrence around the equator in the late declining to minimum phase of the solar cycle (SC). This implies a persistent southward shift of the HCS at these times, a property which has been called the bashful ballerina.

This pattern was later confirmed for several earlier cycles before satellite observations, using HMF sector information extracted from ground-based magnetic field observations (Hiltula $\&$ Mursula 2006). Accordingly, the dance of the bashful ballerina has been verified by Earth-based observations for cycles 16-22. Measurements of the photospheric magnetic field, together with the potential field source surface (PFSS) model

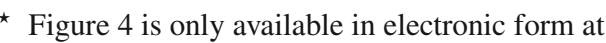
http://www . aanda.org
}

have shown (Zhao et al. 2005) that the average area was indeed larger and the field intensity smaller in north than in south during roughly three years in the late declining to minimum phase of cycles 21-22.

Here we study the HMF and its north-south asymmetry during the exceptional solar cycle 23 (Smith \& Balogh 2008; McComas et al. 2008). We use OMNI HMF observations and Wilcox Solar Observatory (WSO) HMF predictions (Hoeksema 1991) to study the occurrence and latitudinal variation of HMF sectors around the ecliptic/solar equator, and present a new method to study the north-south asymmetry by comparing the $\mathrm{HMF}$ radial field at $1 \mathrm{AU}$ and in the corona.

\section{HMF sectors and HCS asymmetry in OMNI data}

We first study the HCS structure using hourly OMNI-2 data which include in situ HMF observations at 1 AU since 1960s. As earlier (Mursula \& Hiltula 2003), we divide the hourly HMF vectors in two sectors, the $T$ sector (magnetic south) consisting of field lines directed toward the Sun, or the $A$ sector (magnetic north) away from the Sun. We use here the plane division to define HMF sectors (e.g., $T$ sector in GSE coordinates: $B x>B y$ ) but the quadrant division ( $T$ sector: $B x>0$ and $B y<0$ ) yields same results. The total number of $T$ and $A$ sector hours was calculated for each 3-month season around the two high-latitude 


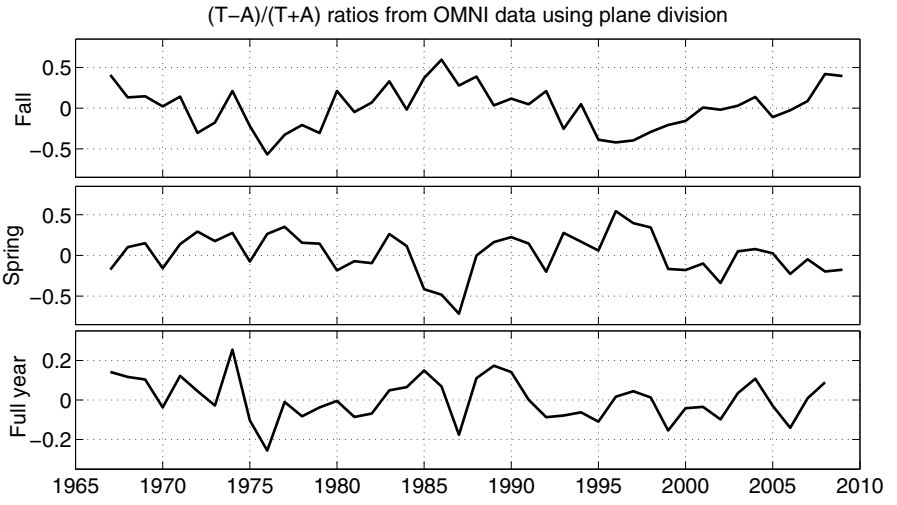

Fig. 1. Seasonal/annual $(T-A) /(T+A)$ ratios in 1965-2008/2009 from OMNI-2 data set using plane HMF sector division. top: fall months (north); middle: spring months (south); bottom: full years.
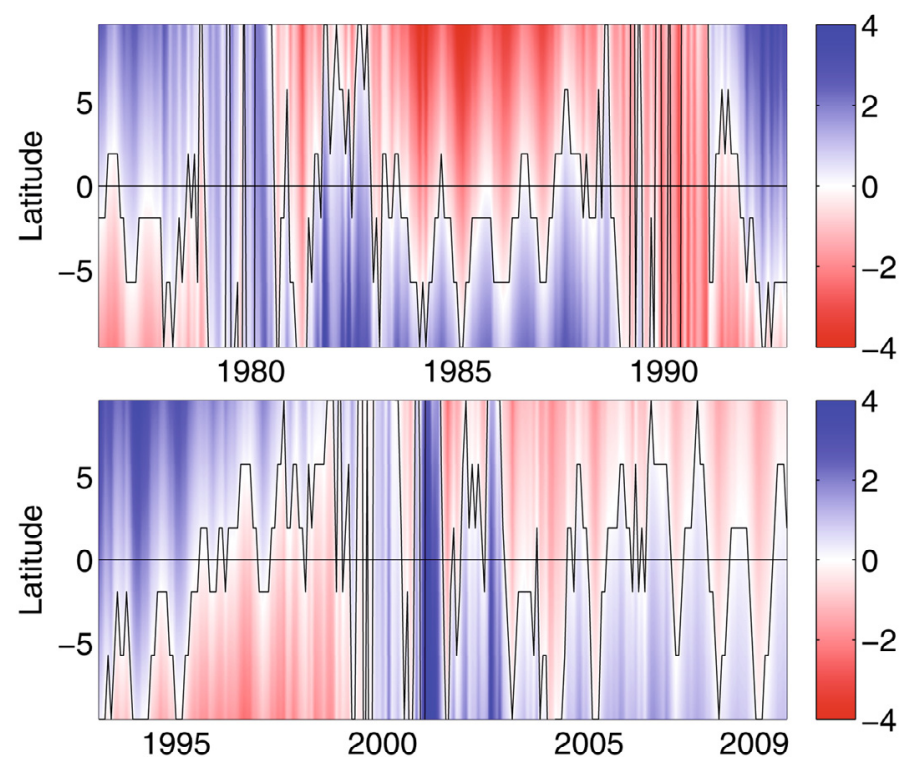

Fig. 2. WSO prediction of coronal radial field (in $\mu$ Tesla) using PFSS model with source surface distance at 2.5 Rs. Magnetic north $(A$ sector) is depicted in blue and south ( $T$ sector) in red. Data were averaged over one solar rotation. Only values around equator are shown. Variable black line denotes the location of field minimum as a proxy of the rotationally averaged HCS. Straight horizontal line denotes the equator.

intervals (Spring $=$ Feb. - Apr.; Fall = Aug.-Oct. $)$ for each full year.

Figure 1 depicts the relative occurrence difference of the two sectors $R_{T A}=(T-A) /(T+A)$ for each Fall (north), Spring (south) and full years. (Analogous results until 2001 were given in Figs. 1 and 2 of Mursula \& Hiltula 2003). The 22-year variation of the ratio around zero depicts the well known pattern of the latitudinal variation (Rosenberg \& Coleman 1969) (RC rule), with the $A$ ( $T$, resp.) sector dominating in Fall (Spring) during positive polarity minima and the $T(A)$ sector in negative minima. However, the RC rule is more systematically developed in Fall where the average 22-year amplitude, prior to 2001, was about 0.16 , implying that the average ratio between the dominant and subdominant sector occurrences around solar minima is 1.94 (Mursula \& Hiltula 2003). In Spring, the average amplitude before 2001 was about 0.11 , making the dominant sector appear only $56 \%$ more often.
Figure 1 shows that the $R_{T A}$ ratio in Fall has reached a strongly positive value in 2008, re-establishing for SC 23 the latitudinal variation (dominance of the $T$ sector now) in north. The $R_{T A}$ value in 2008-2009 is quite typical for previous cycles, almost at the same level (but oppositely signed) as in mid-1990s, but slightly smaller than the largest ratios in mid-1970s and mid1980s. However, the southern hemisphere has, by Spring 2009, not yet developed the normal latitudinal ordering. Note that in earlier minima, the latitudinal ordering in Spring was established in either the same or next calendar year as in Fall. These results suggest that SC 23 is very exceptional in that the RC rule is abnormally delayed or completely broken in the south.

The stronger 22-year variation in Fall leads to the overall annual dominance of the magnetic sector of the north in the declining phase of the solar cycle (Mursula \& Hiltula 2003). This is seen in the annual $R_{T A}$ ratios (Fig. 1, bottom panel) which give the yearly averaged hemispheric asymmetry at 1 AU. Prior to 2001 there is a statistically significant 22 -year baseline oscillation in the yearly $R_{T A}$ ratio due to the dominance of the $A$ ( $T$, resp.) sector in 1970s and 1990s (1960s and 1980s). The $R_{T A}$ amplitude of about 0.09 implies that the HMF sector of the north appears at the ecliptic about $20 \%$ more often around solar minima than the sector of the south (Mursula \& Hiltula 2003).

However, Fig. 1 shows that there is no similar, equally systematic dominance of the $T$ sector in the declining phase of SC 23. There is a period of the expected $T$ sector dominance in 2003-2004 but this is interrupted by A dominance in 20052006 . Thus, the yearly averaged $R_{T A}$ ratios alternate sign fairly regularly and do not show a persistent $T$ sector dominance of at least three years. Since the HCS southward shift has always developed by the time of the maximum of the $\mathrm{RC}$ amplitude in Fall, the three year period of $T$ sector dominance should have developed by the end of 2008. Accordingly, the HCS evolution in SC 23 does not seem to follow the same pattern as in cycles 1622.

\section{WSO coronal field around equator}

Synoptic observations of the photospheric magnetic field together with the PFSS model are used to derive (or "predict") the coronal magnetic field at the source surface distance. Figure 2 shows the Wilcox Solar Observatory source surface radial field at low latitudes averaged over one solar rotation. (We use here the radial model with SS at $2.5 \mathrm{Rs}$ ). Persistent uniform magnetic fields with opposite polarities are seen on either side of the equator during several years around solar minima, changing their polarities from one minimum to another according to the well known 22-year magnetic cycle. On the other hand, around solar maxima the field polarities fluctuate more quickly.

Figure 2 also shows the location of the field minimum, the rotationally averaged source-surface neutral line (SSNL; the coronal counterpart of HCS), as a black line around which the colors are very weak. (PFSS model requires that SSNL/HCS is a zero field surface). Color (field) intensity strengthens when moving away from this line, indicating strong latitudinal gradients around the SSNL/HCS. (Note also that Fig. 2 depicts annual SSNL fluctuations especially around solar minima. This is an artefact due to Earth's annual vantage point variation).

During the uniform polarity times the (yearly averaged) SSNL/HCS is quite stable but there are intervals of a few years when its location is systematically shifted below zero, i.e., towards south. At these times (the bashful ballerina intervals) the HMF sector prevalent in the north (A sector during positive polarity minima and $T$ sector during negative polarity minima) is 


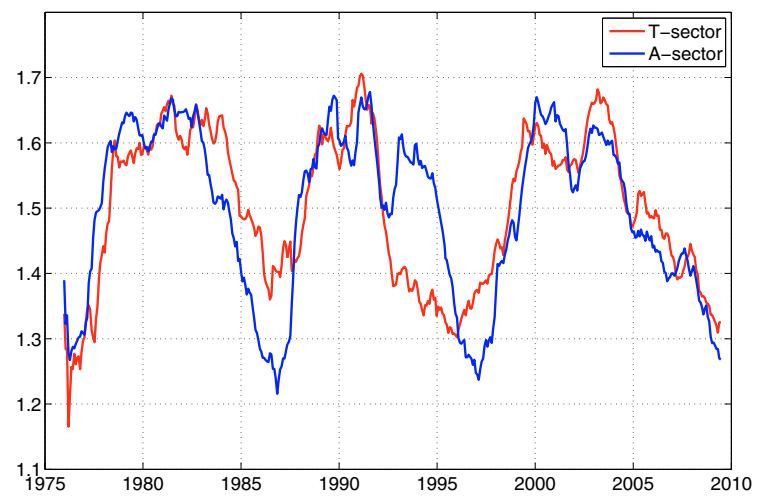

Fig. 3. One year running means of the 9-hourly $n$-values describing the radial decrease of the HMF radial field from source surface to 1 AU. A sector values are in blue and $T$ sector in red.

dominant around the equator. This development is particularly clear in 1976-1977, 1983-1986 and 1992-1994. The same development is also visible in HMF (Fig. 1, bottom panel).

However, no similar continuous 3-year interval of southward shifted SSNL/HCS is found in the declining phase of SC 23. There is a shorter interval in 2003-2004 when the field of north ( $T$ sector) is dominant, but this interval is interrupted by an intrusion of south field past the equator in 2005-2006. Thereafter, since 2008 the northern field is again weakly dominant at the equator. All these developments correspond very well to the OMNI HMF observations (see Fig. 1, bottom panel).

\section{OMNI-WSO comparison of radial HMF}

Let us now study the radial development of the radial HMF field from the source surface to $1 \mathrm{AU}$. We trace the 9-hourly averaged $\mathrm{HMF}$ field (corresponding to $5^{\circ}$ WSO azimuth resolution) observed at 1 AU radially inward to its coronal line-of-sight footpoint at the source surface. We use the observed solar wind speed and its theoretical radial profile (Cranmer 2004) between SS and 1 AU to obtain the delay between $1 \mathrm{AU}$ and corona and the longitude of the solar wind SS source.

According to Maxwell equations, the radial component of the magnetic field should decrease like $B_{r}=B_{0}\left(r_{0} / r\right)^{n}$ with $n=$ 2 . Figure 3 depicts the yearly running means of the 9-hourly $n$ values calculated separately for radially outward ( $A$ sector) and inward ( $T$ sector) HMF values. One can see that the (smoothed) $n$-values are always smaller than 2 . This is mainly due to the PFSS model which requires that the field is zero at SSNL/HCS. Because the line-of-sight footpoints of the HMF observed at $1 \mathrm{AU}$ are often close to model SSNL/HCS where the field is excessively small, this leads to excessively small $n$-values. However, since we are interested in relative differences in the $n$-values only (see later), this bias in the absolute level is harmless and the related latitudinal variation is even advantageous for our present aims.

Accordingly, the $n$-values measure the proximity of the HMF footpoint to the model SSNL/HCS, which mainly depends on the tilt angle and therefore varies with the cycle. Figure 3 also shows that the $n$-values have a significant solar cycle variation. Around solar maxima when the tilt is large, the footpoint is mostly further away from the zero field of the SSNL/HCS, leading to larger footpoint field and larger $n$-values. During solar minima the reverse is true: the footpoint field is, on an average, closer to SSNL/HCS and therefore weaker, leading to smaller $n$-values.

Most interestingly, Fig. 3 shows differences between the $n$ values of the two magnetic hemispheres. The $n$-values of the north are systematically larger than those of the south during the bashful ballerina years of 1983-1986 and 1992-1994 (and partly in 1970s). This is because, at these times, the SSNL/HCS is shifted southward and the northern footpoint is located, on an average, further away from SSNL/HCS than the southern footpoint, thus leading to larger $n$-values in the north. Comparing to Figs. 1 and 2, the new method using the $n$-values depicted in Fig. 3 probably gives the most clear and convincing depiction of the bashful ballerina intervals. We also note that the two major bashful ballerina intervals started soon after a sudden, major decrease of the heliospheric tilt in late 1982 and early 1992 (see the Appendix Fig. 4). When the tilt angle gets smaller, the proximity of the HMF field at $1 \mathrm{AU}$ increases, and the possible hemispheric asymmetry in $n$ gets more easily detectable.

Turning to SC 23, note that the HCS tilt angle has been exceptionally large during the entire declining phase. This is likely related to the exceptionally weak polar fields in SC 23 (Smith \& Balogh 2008) which imply a weak development of latitudinal gradients. As noted above, a large tilt angle tends to mask the hemispheric differences in $n$. Thus, the $n$-values of the two hemispheres are closer to each other in cycle 23 than in the two previous cycles. However, although the conditions in SC 23 are unfavorable for the detection of hemispheric differences from low latitudes, the $n$-values are still larger in the north than in the south in 2003 and again since 2005. This proves that the global field attained the same asymmetry as in the earlier since cycle 16 , although the poor visibility of the unipolar regions at the lowlatitude view point reduced the size of the measured asymmetry. This result is in a good agreement with the observations of the Ulysses probe during its third fast latitude scan in 2007 (Virtanen and Mursula, submitted to J. Geophys. Res. 2010) which show that the high-latitude field was weaker in the north than in the south at that time. Ulysses observations also verify that the HCS region was much wider (about $35^{\circ}$ ) in 2007 than in 1994-1995 (about $20^{\circ}$ ).

Interestingly, the northern $n$-values are larger even in 20052006 when OMNI and WSO data show, in seeming contradiction, that the $A$ sector dominated then around equator. The $A$ sector dominance in OMNI and WSO is related to the occurrence of coronal holes $(\mathrm{CH})$ of northern magnetic ( $A$ sector) polarity at low southern latitudes at this time, increasing the relative occurrence of this polarity at low latitudes temporarily. Low-latitude $\mathrm{CHs}$ are typical for solar maxima and their occurrence in the late declining phase also demonstrates the exceptional nature of SC 23 (see, e.g., Gibson et al. 2009). In addition, polar coronal holes develop slowly in SC 23 and are smaller than in SC 22 (Harvey \& Recely 2002; Kirk et al. 2009), leading to a larger tilt and wider HCS region. Also, the northern polar $\mathrm{CH}$ develops faster than the southern, the relative difference being large around 2005 (Kirk et al. 2009). Accordingly, the dominance of the $A$ sector in OMNI and WSO around 2005, which breaks the development of the bashful ballerina in Figs. 1 and 2, is special feature of SC 23. Therefore, only the $n$-value method can depict the correct situation of the hemispheric difference at this time.

Finally, we note that the present observations give an interesting possibility to interpret the long-term history of solar hemispheric asymmetry. Although HMF sector information exists only since 1930s, the hemispheric asymmetry in solar wind (streamer belt) has been studied since 1840s using geomagnetic activity (Mursula \& Zieger 2001; Mursula 2007). A large 
asymmetry was found during the active cycles in mid-19th century and since 1930s, but during the low-activity cycles around the turn of the 19th and 20th century the asymmetry was weak. This indicates a profound connection between the overall solar activity and the visibility of the hemispheric asymmetry when viewed from low latitudes. The weaker solar activity leads to weaker polar fields, smaller polar coronal holes with unipolar fields, and larger HCS tilts and widths. Since the global asymmetry is the property of high-latitude unipolar fields, this situation strongly reduces their occurrence around the ecliptic. On the other hand, the observed long-term asymmetry indirectly proves that the weak solar activity indeed leads to a considerably wider HCS region around the solar equator.

Moreover, it was found (Mursula \& Zieger 2001; Mursula 2007) that the streamer belt asymmetry experienced a change in orientation between the asymmetric intervals of the mid-19th century and the late 20th century, suggesting a new form of oscillation in the global solar magnetic field with a period of about 200-300 years. This may be the 205-210-year long deVries cycle which appears in many cosmogenic isotope series (see, e.g., Raspopov et al. 2005). Since the streamer belt asymmetry and the HCS asymmetry are most likely related, this implies that the HCS asymmetry in the mid-19th century was opposite to the present orientation, and will again change its orientation in future. So we can predict that, after a period of weak cycles which probably started with SC 23 , the asymmetry becomes more visible at 1 AU again when active rises, but the asymmetry would be then opposite to the present orientation so that the HCS would be shifted northward. Thus, the bashful ballerina would have danced during SC 23 her last dance for some 100-150 years.

\section{Summary}

The global solar magnetic field has long been hemispherically asymmetric so that the field in the north is weaker and has a larger area than in the south (Crooker et al. 1997; Smith et al. 2000; Mursula \& Hiltula 2003; Zhao et al. 2005). We have studied the global HMF during solar cycle 23 using HMF observations at $1 \mathrm{AU}$ and WSO predictions for the coronal field. We found that the latitudinal development of HMF is exceptional in SC 23: while the typical latitudinal variation was attained in the north in 2008, it did not take place in the south until Spring 2009, implying that the Rosenberg-Coleman rule is abnormally delayed or broken for the first time in the satellite era.

We developed a new method to study the hemispheric asymmetry by using the power $n$ of the radial decrease of the radial field from the coronal source surface to $1 \mathrm{AU}$, which gives a measure of the effective (relative) distance of the footpoint to the current sheet. The $n$-values calculated for the two magnetic hemispheres verify the northern dominance in cycles 21-22. There is a similar asymmetry in the high-latitude fields even in SC 23, although it is seen as considerably smaller in low-latitude observations because of the large tilt angle and weak polar fields leading to a wide HCS region. We showed that the $n$-values give a more detailed method to study global asymmetries (bashful ballerina intervals) than the in situ HMF observations or WSO predictions.

The development of SC 23 can help understanding the long-term variation of solar magnetic field and its asymmetry. Hemispheric asymmetry in solar wind (streamer belt) was large during high solar activity in the mid-19th century and since 1930s, but weak during the low-active cycles at the turn of the 19th and 20th century, indicating a profound connection between solar activity and the visibility of the asymmetry at low latitudes. Weaker solar activity leads to weaker polar fields, smaller polar coronal holes and larger HCS tilts and widths. Since the global asymmetry is the property of high-latitude unipolar fields, this situation strongly reduces their occurrence at low latitudes. Note that this evolution indirectly proves that weak solar activity indeed leads to a wider HCS region around the solar equator.

The 200-year variation in asymmetry suggests that solar activity and hemispheric asymmetry will, for some time after SC 23, be less visible at $1 \mathrm{AU}$ but then, with increasing activity, become more visible at 1 AU again. However, the asymmetry will be opposite to the present orientation, with a northward shifted HCS, and larger areas but weaker intensities in the south. Thus, the ballerina would not be bashful for some $100-150$ years.

Acknowledgements. The research leading to these results has received funding from the European Commission's Seventh Framework Programme (FP7/20072013) under the grant agreement no. 218816 (SOTERIA project, www. soteria-space.eu). K.M. acknowledges the financial support by the Academy of Finland to the HISSI research project no. 128189. We are also grateful to NSSDC for OMNI data at http://nssdc.gsfc.nasa.gov/omniweb/. Wilcox Solar Observatory data used in this study and and Fig. 4 were obtained via the web site http: //wso.stanford. edu, courtesy of J. T. Hoeksema.

\section{References}

Cranmer, S. R. 2004, Am. J. Phys., 72, 1397

Crooker, N. U., Lazarus, A. J., Phillips, J. L., et al. 1997, J. Geophys. Res., 102, 4673

Gibson, S. E., Kozyra, J. U., de Toma, G., et al. 2009, J. Geophys. Res., 114, 9105

Harvey, K. L., \& Recely, F. 2002, Sol. Phys., 211, 31

Hiltula, T., \& Mursula, K. 2006, Geophys. Res. Lett., 33, 3105

Hoeksema, J. T. 1991, Adv. Space Res., 11, 15

Kirk, M. S., Pesnell, W. D., Young, C. A., \& Hess Webber, S. A. 2009, Sol. Phys., 257,99

McComas, D. J., Ebert, R. W., Elliott, H. A., et al. 2008, Geophys. Res. Lett., 35,18103

Mursula, K. 2007, Adv. in Space Res., 40, 1034

Mursula, K., \& Zieger, B. 2001, Geophys. Res. Lett., 28, 95

Mursula, K., \& Hiltula, T. 2003, Geophys. Res. Lett., 30, 220000

Raspopov, O., Dergachev, V., Kozyreva, O., \& Kolström, T. 2005, Mem. Soc. Astron. Italiana, 76, 760

Rosenberg, R. L., \& Coleman, Jr., P. J. 1969, J. Geophys. Res., 74, 5611

Simpson, J. A., Zhang, M., \& Bame, S. 1996, ApJ, 465, L69

Smith, E. J., \& Balogh, A. 2008, Geophys. Res. Lett., 35, 22103

Smith, E. J., Jokipii, J. R., Kóta, J., Lepping, R. P., \& Szabo, A. 2000, ApJ, 533, 1084

Zhao, X. P., Hoeksema, J. T., \& Scherrer, P. H. 2005, J. Geophys. Res., 110, 10101

Page 5 is available in the electronic edition of the journal at http://www.aanda.org 
Maximum Inclination of the Current Sheet (N-S Mean) : 1976-2009

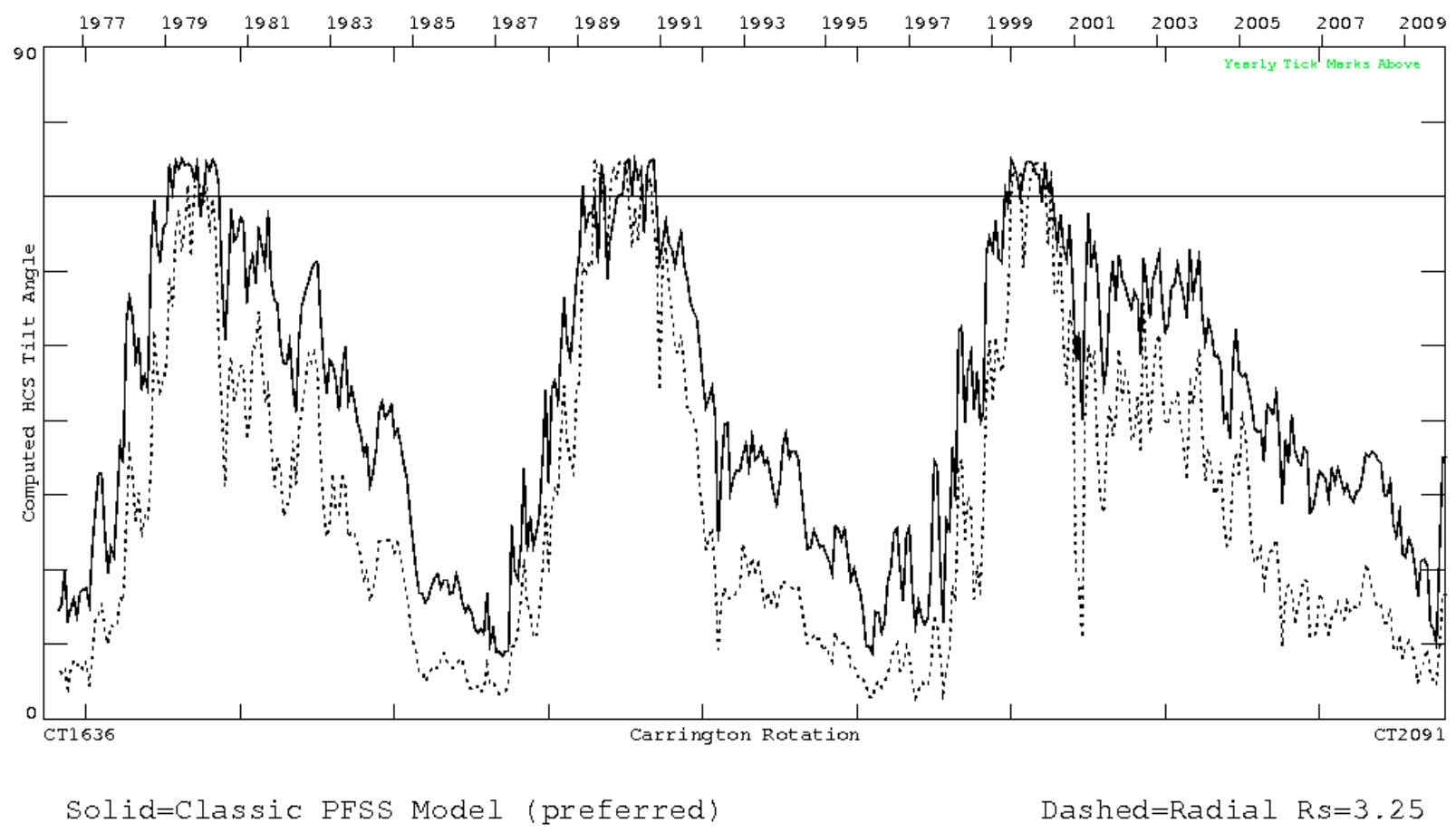

Fig. 4. The maximum inclination (tilt) of the HCS according to WSO PFSS model in 1976-2009. 